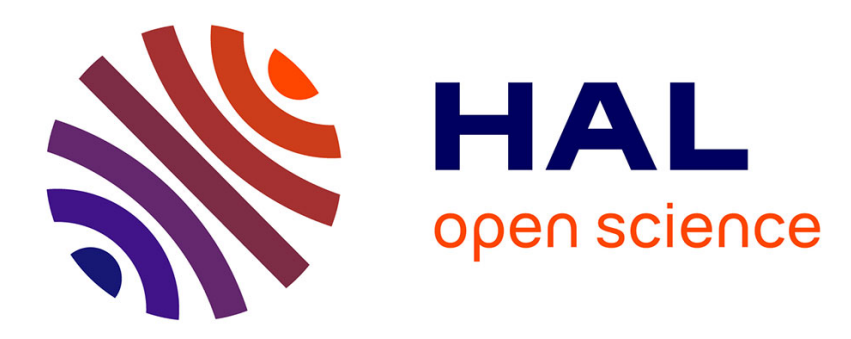

\title{
Toward the monitoring of the spinal cord: A feasibility study
}

Sylvain Feruglio, Olivier Tsiakaka

\section{To cite this version:}

Sylvain Feruglio, Olivier Tsiakaka. Toward the monitoring of the spinal cord: A feasibility study.

Microelectronics Journal, 2018, 10.1016/j.mejo.2018.01.026 . hal-01782182

\section{HAL Id: hal-01782182 \\ https://hal.sorbonne-universite.fr/hal-01782182}

Submitted on 1 May 2018

HAL is a multi-disciplinary open access archive for the deposit and dissemination of scientific research documents, whether they are published or not. The documents may come from teaching and research institutions in France or abroad, or from public or private research centers.
L'archive ouverte pluridisciplinaire HAL, est destinée au dépôt et à la diffusion de documents scientifiques de niveau recherche, publiés ou non, émanant des établissements d'enseignement et de recherche français ou étrangers, des laboratoires publics ou privés. 


\title{
Toward the monitoring of the spinal cord:
}

\author{
A feasibility study \\ O. Tsiakaka ${ }^{a}$, S. Feruglio ${ }^{\text {a, * }}$ \\ a Sorbonne Université, UPMC Univ Paris 06, IFD, CNRS, UMR7606, Laboratoire d'Informatique de Paris 6 (LIP6), IFD, \\ 4 place Jussieu, 75252 PARIS cedex 05, France \\ *Corresponding author: sylvain.feruglio@upmc.fr
}

\begin{abstract}
The usual imaging techniques providing only limited information, there is an unmet need for methods, firstly, to monitor in real time the functional status of the Spinal Cord (SC) and, secondly, to assess the functional consequences of the SC Injuries (SCI) and the effect of therapeutic interventions. To meet these needs, our project aims to investigate a new imaging approach, through the realization of a device for measuring the SC activity. This approach will establish locally and specifically the functional state of the SC in real time, which will provide a breakthrough in surgery, as well as in the care and therapeutic trials for SCI.
\end{abstract}

This paper presents the first results of this research project on pig subjects, focusing on the opto-electrical part, based on Near-InfraRed Spectroscopy (NIRS). Firstly, the pig SC optical characterization was performed in different conditions, using a portable spectrophotometer in order to define the wavelengths of interest and their corresponding optical attenuation. In transmission mode, the Optical Density (OD) was evaluated between 3.5 and 6.5 in the [500; 950] $\mathrm{nm}$ range. In reflection mode, a figure between $11 \%$ and $33 \%$ is obtained in the same wavelength range. We find that wavelengths between $600 \mathrm{~nm}$ and $940 \mathrm{~nm}$ are good candidates to monitor the SC functional activity. Secondly, thanks to these results, a specific opto-electrical system has been designed for the transmission mode only, with adapted light sources and custom probes with its front-end to observe the autonomic functions in the SC. 
Results on the measured haemodynamic variations, at rest and under stimulation, show in real time the impact of a global stimulus on a local section of the SC. However, with a low AC-toDC ratio (around $1 \%$ ), the SC PhotoPlethysmoGram (PPG) acquisition isn't simple and the best trade-off between power consumption and Signal-to-Noise Ratio (SNR) must be found in the perspective of the Embedded System (ES) development.

This study demonstrates, for the first time, the feasibility of the SC activity monitoring using NIRS in transmission mode in a big animal model, where the perfect alignment of the light emitter and receptor isn't necessary due to the diffusive property of the biological media. It contributes to heading towards the use of the Internet of Things (IoT) for medical applications, through the monitoring of the SC during highly invasive processes, such as the stabilization of the spine, in the form of implants and other surgeries, such as that of the aorta, with the use of specific minimally invasive catheters.

Keywords: Embedded system, in vivo experiment, medical device, monitoring, NIRS, optoelectronic, pig model, PPG, spinal cord.

\section{Introduction}

The Spinal Cord (SC) is a major organ composing, with the brain, the central nervous system (CNS). Trauma of the SC (TSC) and pathologies of the spine are major causes of mortality and disability, representing a real public health problem. Their incidence is 28,000 new cases per year throughout the European continent and 12,000 for the USA [1]. For France, there are about 1,200 cases of vertebro-medullar injuries per year [2]. In the orthopaedic and trauma surgery department of the Pitié-Salpêtrière hospital, nearly 50 surgeries are performed each week [3]. After the acute phase, secondary mechanisms, 
including vascular, impact the functional prognosis. Thus, before, during and after the spine stabilization by surgery, the SC status must be observed.

Concerning the particular case of the medullary infarctions, the aortic surgery comes at the forefront of the surgical causes [4-11]. This risk exists in the surgery of coarctation of the aorta, the thoracic aorta dissections, but the most important is the surgery of the aortic aneurysms (after 60 years old, the frequency of the disease in the French population is of 4 to $8 \%$ for men). The risk of paraplegia is evaluated globally at $5 \%$. However, it can reach 30.6 $\%$ in surgical surgery of extensive aneurysms involving the entire descending thoracic aorta and abdominal aorta [10-11]. Thereby, loss of blood flow (ischaemic episode) during thoracoabdominal surgeries increases the risk of somatosensory disorders, in addition to direct damages to the SC. It is therefore essential to be able to observe the activity of the SC in a minimally invasive way during this surgical procedure, without disturbing the surgeon and his team.

To observe the Spinal Cord (SC) and its status, many techniques are available:

1) The well-known Magnetic Resonance Imaging (MRI), which is a non-invasive imagery, is essential for the assessment of lesions at the acute stage and to seek complications at the chronic stage. However, it does not provide functional information (only structural), which is crucial to judge of the SC status. It is the same for myelography, which is a scanner with injection of a contrast media, or the $\mathrm{O}-\mathrm{arm}{ }^{\circledR}$ system of Medtronics, Inc., which requires Xrays use.

2) Functional MRI (fMRI) of the SC is a promising method under development. However, it faces significant limitations. The temporal resolution of fMRI is modest and the spatial resolution is limited by artefacts associated with inhomogeneous magnetic properties of the spine and the physiological movements, including breathing [12]. Moreover, as all non- 
mobile devices, even if such examination can be repeated, it does not provide continuous information. In addition, it imposes a total immobility of the subject. We are in remote conditions for normal living conditions of the subject. To finish, during the surgery act, its employment is not really possible (very high-cost material located in a dedicated room, obligation of the IRM compatibility of the tools in its field of action, etc.).

3) On the functional assessment plan also, the electrophysiological techniques, such as SomatoSensory Evoked Potentials (SSEP) or Motor Evoked Potentials (MEP), are useful as well for per-operative assessment of medullar impairment and they are also commonly used for intra-operative monitoring. During the SC surgery, intra-operative neuromonitoring is assessing the placement of pedicle screws. Misplacement of these screws can lead to lesion of SC or nerve roots that can impair the patient outcome (paralysis, pain, etc.) [13-22]. These techniques, with high temporal resolution as compared to MRI, are invasive, sometimes painful, and currently inappropriate in monitoring medullar function in daily living condition, in its actual form. Moreover and mostly, these techniques, well employed to identify some problems in surgery, cannot identify early the metabolic dysfunction (vascular phenomenon, specially) before the neurological functions are permanently degraded [4-6], which is a serious handicap.

4) Echo-Doppler is also an interesting non-invasive technique. However, the obtained data are rather qualitative. Indeed, only the plethysmograph on a large zone is obtained [23]. Moreover, the SC is easily analysed in newborns through the incompletely ossified posterior arches. After three months, it is more difficult [24].

5) Only recently, the SC is also studied by NIRS. However, it is usually realized with classical intra-operative monitoring equipment, which is not a specifically adapted system and it does not allow chronic measurement $[4-6,11,25]$. In particular, for accurate monitoring of the SC 
activity, percutaneous applications, such as proposed by [6], seem not relevant with the presence of numerous adjacent irrigated tissues (skin, muscle, etc.) that disturb the data measurement in the area of interest and, as for echo-Doppler, the covered area remains wide and shallow. In addition, in current experimental works, it is generally the small animal which has been used as a model [27-30]. Other organs (less restrictive at several levels) are also monitored by this process, with "miniaturized" systems [31-34]. Very recently, a study [26] has shown the feasibility of the SC monitoring in the pig model with a rudimentary custom NIRS system in reflective mode associated to a conventional Mean Arterial Pressure (MAP) system. As for [11], it works only by reflection, which limits the study area to the surface region.

Thus, there is a real unmet need for methods in order to: 1) monitor the haemodynamic status of the SC during various surgeries [4-22], and 2) assess the functional consequences of TSC and, as part of clinical trials, the effect of surgical strategies, pharmacologic, or appropriate rehabilitation $[12,33-39]$. In this project, we aim to contribute to these aspects, proposing a device for continuously measuring various metabolic parameters at the SC level invasively or not, depending on the application context. In both cases, as shown in Fig. 1, the device will employ the Diffuse Optical Imaging (DOI) principle, which is a particular case of Near-InfraRed Spectroscopy (NIRS), and will be implemented using a minimum of one photodetector and two pulsed light sources at different wavelengths, with the associated electronic, for the acquisition of information about, at least, two forms of haemoglobin in realtime. After an elementary processing, the obtained data could be transmitted out of the body for in-depth analysis.

This paper presents the first results of this research project in the Internet of Things (IoT) scope for the biomedical applications context, focusing on the opto-electrical part. Current imaging techniques on organs take advantage of the knowledge we have acquired. Concerning 
the CNS, the brain is well studied since decades. However, so far, few information on the SC functioning can be found. In this paper, to fill in this lack, we firstly propose the pig SC optical characterization in different conditions, and, in a second phase, the use of these results to determine to what extent NIRS could be used to assess neural activity in the spine. This study demonstrates, for the first time, the feasibility of the SC activity monitoring in real-time using NIRS in transmission mode in a pig model, which opens the way for low cost smart embedded medical devices, employing electronics and optics, during and after the surgical act.

This paper is organized in five parts. After a proper introduction, the DOI principle is exposed in section 2. Then, in section 3, our methodology is presented, followed by the experimental results, proposed and discussed. Finally, section 5 concludes this paper proposing an assessment of our contribution and overview of the perspectives.

\section{DOI Principle}

The DOI imagery is based on the Beer-Lambert Law (BLL). In NIRS, the modified version of the BLL is generally employed since several non-ideal factors apply. Thanks to that, the monitoring of the haemodynamic variation can be quantified through the PPG observation with various light sources.

\section{a. Theory of the Modified Beer-Lambert Law}

Light absorption in biological tissues is a complex phenomenon to characterize. However, in the spectral range of $[600 ; 1100] \mathrm{nm}$ (also called the NIR window), the absorption of photons is easily detected and can be used to quantify the optical properties of the physiological tissues. As a DOI technique, NIRS does not discriminate the chemical compositions of these multiple layer materials, thus giving uniform information about the 
different absorbers. Therefore, when biological tissues are illuminated with a monochromatic source, the resulting attenuation (ratio between the incident intensity $\mathrm{I}_{0}(\lambda)$ and the transmitted intensity $I(\lambda)$, as illustrated in Fig. 2) at this wavelength $(\lambda)$ is defined by the BLL:

$$
\mathrm{A}(\lambda)=-\log \left(\frac{\mathrm{I}(\lambda)}{\mathrm{I}_{0}(\lambda)}\right)=\sum_{\mathrm{i}} \varepsilon_{\mathrm{i}}(\lambda) \mathrm{c}_{\mathrm{i}} \mathrm{l}
$$

It depends on the molar extinction coefficient $\varepsilon_{\mathrm{i}}$ (in $\mathrm{L} \cdot \mathrm{mol}^{-1} \cdot \mathrm{cm}^{-1}$ ) of the each absorbing molecule $i$, its concentration in the material $c_{i}$ (in $L^{-1}$.mol) and the mean pathlength of the photons l (in cm). In Fig. 3, the molar extinction of these substances is presented for the main important chromophores for our application. However, the previous BLL equation has been shown inadequate in the case of living tissues. Indeed, several attempts to take the optical complexity of the material agree in the modification of the BLL. Thus, a modified version is now used, taking into account the absorption and the scattering effects, through the respective coefficients $\mu_{\mathrm{a}}$ and $\mu_{\mathrm{s}}$, in:

$$
\begin{aligned}
& A(\lambda)=\sum_{\mathrm{i}} \varepsilon_{\mathrm{i}}(\lambda) \mathrm{c}_{\mathrm{i}} \operatorname{DPF}(\lambda)+\mathrm{G}(\lambda) \\
& \operatorname{DPF}(\lambda)=\frac{1}{2}\left(\frac{3 \mu_{\mathrm{s}}(\lambda)}{\mu_{\mathrm{a}}(\lambda)}\right)^{1 / 2}
\end{aligned}
$$

where the Differential Path Flow (DPF), expressed by equation (3) [40], accounts for the modification of the straight line source-detector length by the wavelength and the type of tissue. The geometry of the volume is considered through $G(\lambda)$ [41]. However, the use of the previous form of the absorption law needs the optical calibration of the volume. So then, to be applied in various conditions, a differential approach (through $\Delta$ ) is conventionally taken in conjunction with the basic form in the following equation:

$$
\left[\begin{array}{c}
\Delta \mathrm{A}\left(\lambda_{1}\right) \\
\Delta \mathrm{A}\left(\lambda_{2}\right) \\
\vdots \\
\Delta \mathrm{A}\left(\lambda_{\mathrm{n}}\right)
\end{array}\right]=\left[\begin{array}{c}
\varepsilon_{\mathrm{a}}\left(\lambda_{1}\right) \Delta \mathrm{c}_{\mathrm{a}} \mathrm{l} \\
\varepsilon_{\mathrm{b}}\left(\lambda_{2}\right) \Delta \mathrm{c}_{\mathrm{b}} \mathrm{l} \\
\vdots \\
\varepsilon_{\mathrm{n}}\left(\lambda_{\mathrm{n}}\right) \Delta \mathrm{c}_{\mathrm{n}} \mathrm{l}
\end{array}\right]
$$


It has to be noted that for an accurate measurement, one must identify all the chromophores in the medium. As presented in the previous set of equations (4), it is then obvious that $n$ chromophores can only be quantified with at least $n$ light sources. In blood perfused tissues, compounds of haemoglobin are targeted for monitoring, because whole blood is considered as the major absorbing substance. However, it is most importantly because it is the lone time varying component (as described in the following paragraph). The main chromophores are specifically oxyhaemoglobin $\left(\mathrm{HbO}_{2}\right)$ and deoxyhaemoglobin $(\mathrm{Hb})$, although dyshaemoglobins, such as carboxyhaemoglobin $(\mathrm{COHb})$ and methaemoglobin $(\mathrm{MetHb})$ are infamously known to alter the measurement of oxygen saturation in blood [42-43]. Then, the necessity to quantify their concentration arises and, with it, additional light sources are needed.

\section{b. Monitoring of the Haemodynamic Variation}

Illuminated tissues transmit the incident photon flux attenuated by the different layers. At the one-second time scale, the skin, flesh and bones have a static absorbance that is responsible for the DC attenuation part of the output signal, whereas blood induces an alternative modulation of the transmitted (or reflected) signal, namely the AC part. This specificity allows the discrimination of the blood related changes from the rest. The PhotoPlethysmoGraphic (PPG) monitoring is a simple technique to set up in order to assess blood volume changes, assuming the acquisition system provides a sufficient contrast (i.e. AC-to-DC ratio), typically below $10 \%$ [43]. At each contraction of the heart (systole), blood is pumped into the arteries throughout the circulatory system. As the arteries and the microvascular bed are filled, the absorbance of the tissues increases. With the end of the cardiac cycle (diastole), the blood volume in the arteries decreases, as well as the absorbance. The resulting converted signal at the photoreceptor is a time varying signal known as the PPG [43], represented in Fig. 4. 
The origin of this waveform is yet unclear. On one hand, some consider that the blood returning from the periphery to the heart through the veins, as a very low frequency signal, has an additional impact on the total absorbance in the DC part [29]. On the other hand, previous studies relate the pulsatile part of the PPG to the change of diameter of the arteries, arterioles and capillaries, whether by modification of the photon pathlength [41] or pressure induced interactions [44]. Others link the AC part to the evolution of the alignment of the erythrocytes relatively to the luminous flux during the cardiac cycle, modifying the optical scattering [45]. In spite of a consensus on the matter, it remains a promising tool to assess the cardiovascular system.

In the same way as the ElectroCardioGram (ECG) is used to derive some vital signs and to monitor the electrical conduction of the myocardium, the PPG can give information about the volumetric functions of the heart, but also about the circulatory system at the location of the measurement [46-50]. Nonetheless, it still has a way to go before being truly integrated into the healthcare professionals' routine, considering there is an unlimited variety of shapes and characteristics of this waveform, depending on the observation site and among individuals [51]. So far, only pulse oximetry makes a good use PPG monitoring, when multi acquisition with different wavelengths are performed (typically, $660 \mathrm{~nm}$ and $940 \mathrm{~nm}$ ). The AC and DC components of the signals are then separated to compute the oxygen saturation of the blood, also called $\mathrm{SpO} 2$ [43], through the following relation:

$$
\mathrm{SpO2} \%_{\%}=\frac{\mathrm{c}_{\mathrm{Hb}_{2}}}{\mathrm{c}_{\mathrm{Hbo}_{2}}+\mathrm{c}_{\mathrm{Hb}}} * 100=\mathrm{A}-\mathrm{B} \frac{\left.\frac{\mathrm{AC}}{\mathrm{DC}}\right|_{\lambda_{1}}}{\left.\frac{\mathrm{AC}}{\mathrm{DC}}\right|_{\lambda_{2}}}
$$

where $\mathrm{C}_{\mathrm{HbO}_{2}}$ and $\mathrm{c}_{\mathrm{Hb}}$ are the concentrations of $\mathrm{HbO}_{2}$ and $\mathrm{Hb}$, respectively. $\mathrm{A}$ and $\mathrm{B}$ are calibration factors depending on the location of the measurement and the type of subject [43, 51-53]. 


\section{Methodology}

In this section, the material and protocol for these in vivo experiments are presented. It is followed by the instrumentation description.

\section{a. Material and Protocol}

In vivo experiments were performed with veterinaries of the XP-MED society, after obtaining permission from the ethical committee of Centre de Recherche en Imagerie Interventionnelle (Cr2i, APHP - INRA, Jouy-en-Josas, France), under the authorization number 03-1405.

Due to its anatomic proximity with humans, both in structure and vascularization, a FBM (Familial hypercholesterolemia Bretoncelles Meishan) pork subject had been chosen for these experiments. During these later described experiments, adult pigs (about twelve months old) and about forty kilograms have been chosen. As you see through Fig. 5, the dimensions of the vertebral column make it a good fit for tests aiming a potential transfer to human being (diameter close to $1.5 \mathrm{~cm}$ for the $\mathrm{SC}$, encapsulated in multiple layers in the spinal canal of approximately $2 \mathrm{~cm}$, protected by the vertebral body).

Firstly, the subject was pre-medicated with a ketamine injection. Then, the animal was intubated after anaesthesia and a mixture of isoflurane and oxygen maintained the animal under general anaesthesia during all the procedure. Mechanical ventilation was also performed with the Primus anaesthesia workstation from Dräger Medical, Inc. The body temperature measurement was executed in real-time and the oxygen level was continually controlled on its tongue, with the Infinity Gamma XXL of Dräger Medical, Inc.

For the surgery, the animal was positioned on ventral decubitus. An incision was made to expose the vertebral column from T11 to L3, where the anterior part of the blood supply of 
the spine is accessible [54]. On a second phase, subsequently called case 2, a laminectomy on the region of interest was performed.

This study is composed of two highly related in vivo experiments on a pig's spine into two different cases. All observations are performed on the T14 vertebral level. This location is selected because it encompasses the link between the SC and a major segmental medullary artery, the artery of Adamkiewicz. Responsible for the supply of blood in the lower section of the CNS $[54,55]$, a strong correlation exists in human between a damaged Adamkiewicz and the loss of sensorimotor functions [55]. However, taking into account the disparity of biological parameters between two subjects of the same species, in situ characterization must precede biomedical imaging. Hence, the first experiment consists in the optical characterization on the pig SC. Then, the SC haemodynamic variations are measured thanks to the PPG monitoring. Fig. 6 shows the optical path scheme in the vertebra, which the experiments are based on. The emitter (E) and the receiver (R) are in contact with the sides of the near-flat spinous process (around $1 \mathrm{~cm}$ by $1 \mathrm{~cm}$ ), called lamina, close to the pedicle region. Thus, the luminous flux propagates through multiple biological layers of the SC, including especially the vertebral body (bone), fat (in the epidural space), the dura mater, the spinal meninges in the subdural and the subarachnoid spaces, the CerebroSpinal Fluid (CSF), veins, arteries and the white and grey matters. Each layer modulates the spectrum of the input light. For us, only the variations of light due to the SC activity are relevant. They correspond to a neuronal action, oxygen-consuming and visible thanks to the variations of oxygenated and non-oxygenated haemoglobin. After this first case study (case 1), the lamina is suppressed (case 2) to enable the same measurements directly in contact with the dura matter, without the presence of bone.

\section{b. Instrumentation}


Two kinds of instrumentation have been employed: A commercial system for the SC optical characterization and a custom opto-electrical system for the PPG monitoring.

\section{i. Optical characterization of the SC}

The SC is a complex multi-layered structure, where optical reflections are present at each interface and interact with absorption and scattering in each layer. Contrary to the brain, information about optical properties of the SC is sparse. Many papers talk about specific tissues, rather associated to the brain, such as grey matter, dura matter, bones, etc. [57, 58]. Very few papers [58-62] mention optical reflectance in specific conditions. To our knowledge, only one curve of the optical transmittance can be found in the literature [64] for ex vivo rats. The complexity of the in vivo measurement seems to be the principal reason to the lack of data. Moreover, until now, the study of this organ did not show as much interest in the medical world as the brain.

In order to develop an optimized ES, with low power consumption especially, to monitor the SC activity of a pig, thanks to DOI, it is of first importance to quantify the transmittance and the reflectance of the SC in both the visible and NIR ranges. With it, wavelengths of interest (and irrelevant) can be highlighted. Moreover, the order of magnitude of the DC attenuation factor can be quantified, which permits to size the instrumentation chain, with the best trade-off between SNR and power consumption [65].

Spectral characterization of the pig SC has been realized in various conditions with the portable spectrophotometer (AvaSpecULS2048XLUSB2, Avantes Inc.), with a resolution of 3 $\mathrm{nm}$, associated to a white light source (AvaLight-HAL). For the transmittance measurement, two optical fibers (FC-UVIR800-2-ME and FC-UVIR1000-2-ME) are applied to each side of the SC (see Fig. 6), for case 1 and case 2. Concerning the reflectance measurement, it is a reflective fiber probe (FCR-7UVIR200-2-45-ME) that is employed, after calibration with a 
white reference (WS-2). The commercial software AvaSoft has been used to command and to register the data.

Note that in this experiment, unlike the following, the perfect alignment of the both probes is obligatory due to the small diameter of the optical fibers (1000 $\mu \mathrm{m}$ in reception).

\section{ii. PPG monitoring}

In the second part of this study, the DOI technique was applied on the pig SC in order to obtain the SC PPG for a minimum of two different wavelengths in transmission mode. For this purpose, a low cost custom opto-electrical system has been designed. Its synoptic is presented in Fig. 7. It is composed of:

- The microcontroller is a mbed LPC1768 of NXP Semiconductors N. V., connected to the computer for real-time signal observation and power supply in USB 2.0 in one side and to the opto-electrical system in the other side. Chosen for rapid prototyping, its capabilities make a good fit for an IoT device.

- Two different light sources emitted alternatively. This illumination used two LEDs at 640 nm (L-1513SRC-J4, Kingbright Corp.) and $850 \mathrm{~nm}$ (LED851L, Thorlabs, Inc.), respectively. They are pulsed at a frequency of $400 \mathrm{~Hz}$, with an ON state of $50 \mu$ s where $20 \mathrm{~mA}$ are typically delivered on $3.3 \mathrm{~V}$, thanks to two outputs of the mbed in association with resistors. These elements have been chosen for their high illumination level mainly, with a relatively low illumination cone and low cost, at the expense of the very low power consumption and low wavelength resolution in comparison to other possible modern light sources, such as VCSEL for example. Note that the illumination angle must be chosen small in reason of the diffuse nature of the various tissues. This has the advantage of releasing the constraint on the alignment of the optical transmitter-receiver pair and on the directivity of the source. Moreover, we can remark that the $5 \mathrm{~mm}$ LEDs fill most of the available lamina area on the vertebra for emission $(1 \mathrm{~cm} \times 1 \mathrm{~cm})$. 
- The photodector is a BPW34 photodiode of Vishay Intertechnology, Inc. It is selected mainly for its high spectral sensitivity for visible and NIR light and its flat packaging of $5.4 \mathrm{~mm}$ by $4.3 \mathrm{~mm}$ with a relatively large sensitive area of $7.5 \mathrm{~mm}^{2}$, particularly adapted to our application. This photodiode has been supplied with a reverse voltage of $900 \mathrm{mV}$, thanks to the TransImpedance Amplifier (TIA) circuit, in order to optimize the optical responsivity and the response time.

- The associated TIA, converting the photocurrent to a voltage, is based on a low noise precision operational amplifier OPA140, from Texas Instruments Inc., with a feedback metal resistance of $4.7 \mathrm{M} \Omega$ in parallel with a tantale capacitance of $2 \mathrm{pF}$. It is powered by a voltage regulator in $0-5 \mathrm{~V}$. With a rise time $10-90 \%$ of $22 \mu$ s (i.e., bandwidth of 16.9 $\mathrm{kHz}$ ), it allows the observation of fast varying physiological phenomena with optimally reduced oscillations.

- The ADC is an ultra-low noise 24-bits Sigma-Delta type, of reference AD7190, Analog Devices, Inc. This large number of bits was chosen with a prototyping approach in mind. With this high resolution, the conversion of the complete signal is executed in order to obtain both the AC and DC contributions without employing specific analog filters in input and two different conversion channels. The ADC has been configured in order to have a continuous conversion, with an internal gain of 1 , an output date rate of $400 \mathrm{~Hz}$. The $\mathrm{Sinc}^{3}$ chop filter has been disabled to limit the deformation of the spectrum. In this configuration, we have a noise voltage of $4 \mathrm{mV}_{\mathrm{RMS}}$ in the worst case, and an effective resolution close to 19 bits.

- With the exception of the USB-powered microcontroller and LEDs, the overall system runs on a $9 \mathrm{~V}$ alkaline battery with a LM317 voltage regulator delivering $5 \mathrm{~V}$.

Concerning the software part, a custom LabVIEW (National Instruments Corp.) interface is used to command the microcontroller, observe in real-time the waveforms, guarantee the 
probes placement and the good shape of the measurement (the resulting PPG signals detected by transmission process through the spine), and also record data for the off-line postprocessing.

The post-processing is led in a semi-empiric fashion. Since the numerical manipulations are not made on-line, trial and error methods can be applied. Starting from the work of [43], the pulsatile component lies around $1 \mathrm{~Hz}$. Depending on the heart rate, one could restrict its range to $[0.5 ; 5] \mathrm{Hz}$, corresponding to $[30 ; 300] \mathrm{bpm}$. The respiratory modulation is in the [0.13;0.48] Hz window, according to [47]. Thus, all existing frequencies below $0.13 \mathrm{~Hz}$ belong to the DC part of the PPG.

This signal conditioning is performed with a dedicated Python program. Firstly, a Fast Fourier Transform is applied on the samples to verify the aforementioned frequency windows. Spurious signal, due to pseudo-periodic displacement of the spine in the vertebral canal with breathing for example, are taken care of. Indeed, these artefacts have been targeted in a study for a different application of NIRS on the SC [66], whereas here, they are discarded. Then, IIR filter of high order cancel out all contributions above $5 \mathrm{~Hz}$ to reduce intrinsic noise (output measured at $2 \mathrm{mV}_{\mathrm{RMS}}$ ) mainly, since no useful information resides there. The relevant piece of information being in a narrow band with relatively low amplitude for the AC component, the slight delay between frequency components could change the shape of the signal, hence the use of Bessel filter in post-processing. Then, for a separate observation of the DC component and the respiration mainly, the whole $[0 ; 0.48] \mathrm{Hz}$ range is extracted.

\section{Experimental Results}

The experimental results are presented in this section. Following the SC spectral characterization in both transmission and reflection modes, the first proof of concept of the SC PPG monitoring in the big animal is presented. Note that these findings, originating from two distinct experiments, are consistent. 


\section{a. SC Spectral Characterization}

The optical transmittance versus wavelength is presented in Fig. 8 in the range of $500 \mathrm{~nm}$ to $950 \mathrm{~nm}$ before laminectomy (case 1 in Fig. 8 corresponding to thoracoabdominal surgeries) and after (case 2 corresponding to orthopaedic applications). In case 1, the distance between the fibers is then close to $23 \mathrm{~mm}$ and $19 \mathrm{~mm}$ in case 2 . The reflectance can be seen in Fig. 9 . Highly noisy data at lower wavelength than $500 \mathrm{~nm}$ aren't shown, mainly on account of low illumination of the employed light source and the high absorption of this biological environment. Moreover, wavelengths higher than $940 \mathrm{~nm}$ are irrelevant, because water (which corresponds to $77 \%$ of the SC) highly absorbs the illumination flow. Note that, contrary to the measure in reflection, in vivo transmittance measurements are delicate on a so peculiar geometry that is the vertebra.

Concerning the transmittance, the maximum transmittance is $2.810^{-3} \%$, which corresponds to an OD of 3.65. In case 2, the transmittance is then improved tenfold, with a maximum value of $2.410^{-2} \%$ (OD of 4.55). Between 500 and $600 \mathrm{~nm}$, lobes due to oxyhaemoglobin are dimly visible after laminectomy and indistinguishable before. Between 750 and $760 \mathrm{~nm}$, we can see the effect of fat before laminectomy. After the surgical act, the most part of it has been removed. Therefore, its impact is much less pronounced. Finally, around $950 \mathrm{~nm}$, the peak is due to an artefact.

Concerning the reflectance (see Fig. 9), the shape of the curves is very different between both cases. In case 1 , before $600 \mathrm{~nm}$, reflection is close to 0 , in contrast to case 2 , where the haemoglobin presence is notable. This difference is clearly due to the bone. Then, the reflectance increases to reach a maximum close to $25 \%$ in case 1 around $680 \mathrm{~nm}$ and $33 \%$ around $640 \mathrm{~nm}$ in case 2. And, finally, these curves decrease exponentially after with a minimum value close to $11 \%$. 
In the light of these observations, we can say that, in both cases, wavelengths between 600 $\mathrm{nm}$ and $940 \mathrm{~nm}$ are good candidates to monitor the SC functional activity. As the bone highly contributes to absorb light, it is advised to find methods to suppress its bad optical effect for a good observation of the haemodynamic variations and to reduce the light sources power consumption. Moreover, with a maximum depth of penetration of $5 \mathrm{~mm}$ typically for the visible light [67-69], only the SC surface can be observed in reflection mode. Thus, the motor neurons region, situated in the ventral side (see Fig. 6), for example, cannot be monitored. Consequently, for a real SC monitoring, the transmission mode, with a good placement of probes, is the only possibility. The interest of the reflection mode employment is minor.

\section{b. PPG monitoring}

The experimentation proceeds to the PPG monitoring on the aforementioned vertebral column section.

A sample of a dual PPG acquisition in the two configurations is shown is Fig. 10. With respect to Fig. 4, the static offset component is present. The cardiac influence is slightly less visible, because of the large respiratory modulation. Before laminectomy (case 1), the PPGs showed a contrast around $1 \%$ for the red source and only $0.4 \%$ for the InfraRed source. After laminectomy (case 2), it increases up to $1.4 \%$ and $1.7 \%$, respectively. It is not surprising to have such low ratios as the low density of vascularization in the SC does not match that of typical chosen sites, like the finger or the earlobe, as well as the presence of the vertebra in case 1 , influencing the DC value.

With two PPGs simultaneously recorded at different wavelengths, mean values for oxygen saturation can be computed. In case 1, the classical formula gives unusual values, expressed by equation (5) with $\mathrm{A}=110$ and $\mathrm{B}=25$. We suppose that it is due to the complexity of the DPF associated to the high attenuation of the red light in this configuration. In case 2 , the 
value of $93 \%$ is calculated. Relatively to human, this number is abnormally low, warning a hypoxaemia [43]. However, when considering the different calibration for species, there is an error in the direct computation of the oxygen saturation. Applying the correction for the pig $[52,53]$, gives a $\mathrm{SpO} 2$ at $96 \%$. This value is in agreement with the Infinity Gamma XXL readings.

The impact of different stimuli was observed in the experiments. Chemical stimulations were realised with an intravenous injection of a bolus of epinephrine. An example of the systemic response is shown in Fig. 11, where the PPG, monitoring at $850 \mathrm{~nm}$, sees dramatical perturbations. During the recording, the animal was not under artificial ventilation. Few seconds after the injection (visible artefact on the plot), the baseline is modified as the $\alpha$ and $\beta$ effects of the neurotransmitter take place:

- the cardiac output, reflected by the amplitude of the signal, increases by $150 \%$, as a sign of a strengthening blood flow;

- the heart rate raises by about $18 \%$ above the resting value;

- the signal shape shows the stiffening of the vascular structure, as the dicrotic notch flattens $[49,69]$.

As the stimulation effects amplify, the cardiac frequency reaches $41 \%$ of the baseline cardiac frequency. Approximately 2 minutes after the injection, respiratory function was spontaneously re-established. It is easily distinguishable for its large low frequency modulation on the PPG.

\section{Conclusion and Perspectives}

This project proposes to design a highly innovative ES in the biomedical field, responding to real public health problems and complementing systems already used routinely. It gives 
rise to both scientific and technical issues that have not yet been explored or too little. Indeed, up to this date, no published study has shown large animal model results with custom optoelectrical embedded probes, demonstrating the feasibility of this imaging technique on subjects of size comparable to humans for a potential transfer.

In this paper, the first steps of DOI imaging of the SC are presented on a big animal model. Optical characterization has been done with the vertebra and without it. The impact of the bone on the luminous flux has been evaluated and its impact on the power consumption of the future device has been taken into account. In a second part, the first results of DOI imaging have successfully demonstrated the ability of the transmission mode to monitor the haemodynamic variations in the same two configurations.

The proposed application of NIRS for the SC opens the way for the use of new IoT in the medical field. Through the monitoring of the SC during highly invasive processes, such as the stabilization of the spine, the device could be implemented in the form of implant and for other surgeries, such as that of the aorta, with the use of specific minimally invasive catheters for example.

However, in both scenarios, the compactness of the system has to be assured. Consequently, other light sources in a denser package could be used in the future to optimize the volume. Moreover, VCSEL components could help reduce the power consumption of the system without compromising on the measurement. As stated before, the use of a sufficiently large collection area of the luminous flux matches our needs in this application. Nonetheless, an array arrangement of several photodetectors could help for the durability and reliability of the implanted device. These sensors could be CMOS buried multi PN junction photodetectors $[71,72]$ in order to enjoy of their multispectral ability and their rejection of certain parasitic signal and noise. Therefore, an ASIC can be designed. Finally, the addition of other sensors 
(humidity, temperature, pressure, etc.) is possible for a more accurate aid in the diagnosis, safety and security.

\section{Acknowledgement}

This work was performed within the Labex SMART (ANR-11-LABX-65) supported by French state funds managed by the ANR within the Investissements d'Avenir programme under reference ANR-11-IDEX-0004-02.

We thank Dr. Pierre-François Pradat, $\mathrm{PhD}$, and Veronique Marchand-Pauvert, $\mathrm{PhD}$, for discussions.

\section{Bibliography}

[1] National Institute on Disability and Rehabilitation Research, Office of Special Education and Rehabilitative Services, US Depart. of Education, Washington, DC.

[2] http://www.irme.org/.

[3] http://icm-institute.org/.

[4] M. Fok, et al., Proc. of the 8th Intern. Conf. Sensing Technology, 2014.

[5] A. Moerman, et al., J. Endovasc. Ther., 18(1), pp. 91-95, 2011.

[6] C.D. Etz, et al., Europ. J. Vasc. Endovasc. Surg., 46(6), pp. 651-656, 2013.

[7] http://www.yksimed.com/index.php?option=com_comprofiler\&task=viewProfile \&userid=145\&lang=fr.

[8] E. Kieffer, et al., J. Vasc Surg., 35(2), pp. 262-268, 2002.

[9] E. Kieffer, et al., Annals of Vascular Surgery, 22(6), pp. 822-828, 2008.

[10] C. Masson, J. Neuroradiol., 31, pp. 35-46, 2004.

[11] A.S. Kogler, et al., Anesthesiology, 123(6), pp. 1-12, 2015.

[12] P.W. Stroman, et al., Neuroimage, 1(84), pp. 1070-1081, 2014. 
[13] J.M. Hicks, et al., Spine, 35(11), E465-470, 2010.

[14] B. Calancie, et al. , Spine, 17(10), pp. 1229-1235, 1992.

[15] B. Calancie, et al., Spine, 19(24), pp. 2780-2786, 1994.

[16] B. Calancie, et al., J Neurosurg. Spine, 20(6), pp. 692-704, 2014.

[17] A.L. Mikula, et al., J Neurosurg. Spine, 24(4), pp. 624-638, 2015.

[18] C.H. Lee, et al., Clin. Neurophysiol., 126(10), pp. 2019-1025, 2015.

[19] M.L. Donohue, et al., J. Clin. Neurophysiol., 29(6), pp. 502-508, 2012.

[20] D.G. Anderson, et al., Spine, 27(14), pp. 1577-1581, 2002.

[21] R.D. Rose, et al., Spine, 22(3), pp. 334-343, 1997.

[22] M.R. Isley, et al., Neurodiagn. J., 52(2), pp. 100-175, 2012.

[23] N. Nair, et al., The Indian J. of Rad. \& Imaging, 26(4), pp. 493-501, 2016.

[24] http://www.sfip-radiopediatrie.org/SFIPoldpages/EPUTRO02/GUITRO02.HTM.

[25] A.J. Macnab, et al., Spine, 27(1), pp. 17-20, 2002.

[26] B. Shadgan, et al., Proc. SPIE, 100720T, 2017.

[27] B. Shadgan, et al., Proc. SPIE, 100380N, 2017.

[28] J.P. Phillips, et al., J. Physics. Conf. Series 307(1): 012 016, 2011.

[29] J.P. Phillips, et al., J. Biomed. optics, 18(3): 037 005, 2013.

[30] A. Goguin, et al., IEEE Trans. Biomed. Circ. \& Syst., 4(5), pp. 329-335, 2010.

[31] P. Bingger et al., IEEE 23rd Intern. Conf. MEMS, pp. 1031-1034, 2010.

[32] B. Shadgan, et al., Proc. SPIE, 100380I, 2017.

[33] J.M. May, et al., Biomed. Phys. Eng. Express, 3(1), 015023, 2017.

[34] Z. Patel, et al., IEEE EMBS, pp. 1840-1843, 2017.

[35] F. Miyanji, et al., Radiology, 243(3), pp. 820-827, 2007.

[36] M.K. Tewari, et al., Surg. Neurol., 63(3), pp. 204-209, 2005.

[37] A.E. Flanders, et al., Radiology, 177(1), pp. 25-33, 1990. 
[38] D.M. Schaefer, et al., Spine, 14(10), pp. 1090-1095, 1989.

[39] D. Lammertse, et al., J Spinal Cord Med., 30(3), pp. 205-214, 2007.

[40] F. Scholkmann, et al., J. Biomed. Opt, 18(10), 105004-1, 2013.

[41] D.A. Boas, et al., NeuroImage, 13(1), pp. 76-90, 2001.

[42] J.A. Brunelle, et al., Scand. J. of Clin. and Lab. Invest., 56(sup224), pp.47-69, 1996.

[43] J.G. Webster, CRC Press, 1997.

[44] A.A. Kamshilin, et al., Scientific Reports, 5, Srep10494, 2015.

[45] I. Fine, Optical Tech. in Biophysics and Medicine XV; and Laser Physics and Photonics XV, 9031, pp. 903103, 2014.

[46] J. Allen, et al., Physiological meas., 24(2), 297, 2003.

[47] J. Allen, et al., Physiological Meas., 28(3), pp. R1-R39, 2007.

[48] I. Jeong, et al., Yonsei Medical J., 51(3), 345, 2010.

[49] Q. Yousef, et al., Meas. Science Review, 12(6), 2012.

[50] C.C. Wei, IEEE Trans. on Biomed. Eng., 60(1), pp. 151-154, 2013.

[51] J. Allen, et al., Computers in Cardiology, pp. 485-488, 2004.

[52] J.A. Pologe, et al., US Patent 7430 444, 2008.

[53] W. Erhardt, et al., J. of the Assoc. of Vet. Anaesthetists of Great Britain and Ireland, 17(1), pp. 30-31, 1990.

[54] L.G. Svensson, et al., The Annals of Thoracic Surgery, 49(4), pp. 528-536 1990.

[55] J.T. Strauch, et al., The Annals of Thoracic Surgery, 83(6), pp. 2130-2134, 2007.

[56] G. Melissano, et al., E. J. of Vascular and Endovascular Surgery, 38(5), pp.567-577, 2009.

[57] S.L. Jacques, Phys. Med. Biol., 58(11), pp. R37-R61, 2013.

[58] D.A. Boas, et al., CRC Press, New York, 2011.

[59] A.J. Macnab, et al., SPINE, 27(1), pp. 17-20, 2002. 
[60] A.N. Yaroslavsky, et al., Phys. Med. Biol., 47(12), pp. 2059-2073, 2002.

[61] R.R. Gangidi, et al., JFS C: Food Chemistry and Toxicology, 70(6), pp. c397-c400, 2005.

[62] H. Radhakrishnan, et al., J. of Biomed. Optics, 10(5), 051405, 2005.

[63] A.E. Desjardins, et al., Biomed. Opt. Express, 2(6), pp. 1452-1461, 2011.

[64] K.R. Byrnes, et al., Lasers in Surgery and Medicine, 36(3), pp. 171-185, 2005.

[65] K.N. Glaros, et al., IEEE BioCAS, pp. 245-248, 2009.

[66] E.W. Wolf, Wiley Neuromodulation, 18(6), pp. 448-459, 2015.

[67] R.R. Anderson, et al., J. Invest. Dermatol., 77, pp. 13-19, 1989.

[68] G. Strangman, et al., Neuroimage, 18(4), pp. 865-879, 2003.

[69] M.T. Politi, et al., Computers in Biology and Medicine, 72, pp. 54-64, 2016.

[70] S. Chatterjee, et al., Biomed. Phys. Eng. Express, 2(6), 065018, 2016.

[71] S. Feruglio, et al., IEEE Sensor Journal, 16(6), pp. 1611-1620, 2015.

[72] G.N. Lu, et al., SPIE, Applied Optics and Photonics China (AOPC), pp. 1-6, 2015. 


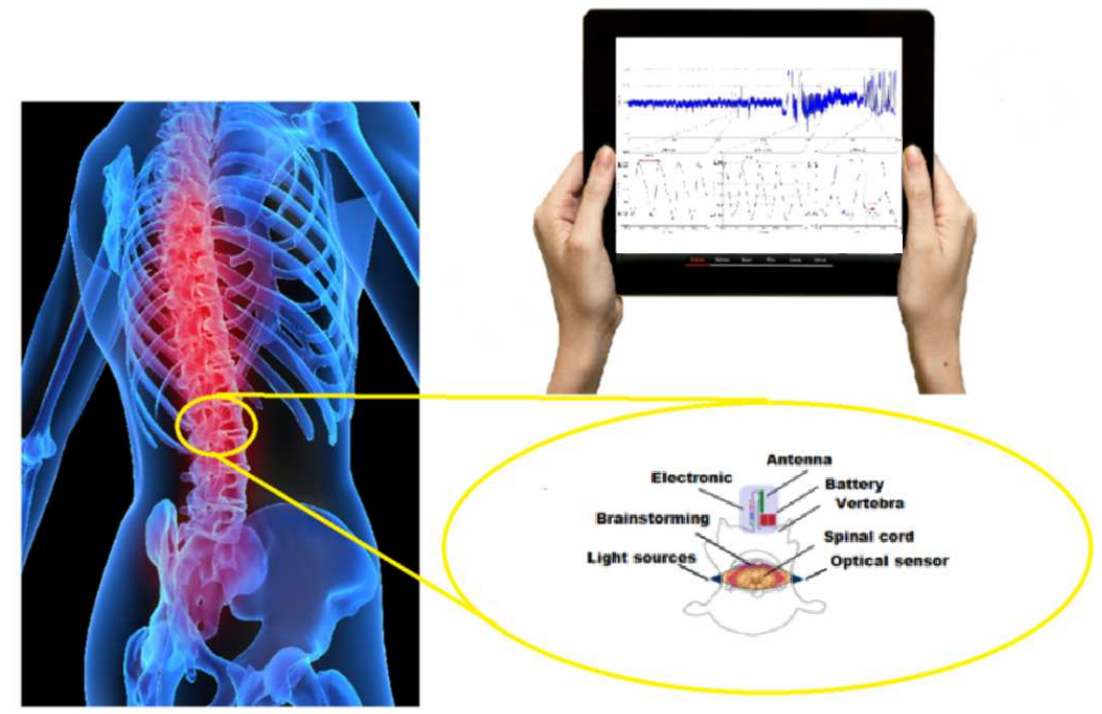

Fig. 1: Illustration of the project in the case of the TSC monitoring. Original image on the left side coming from Wikipedia.

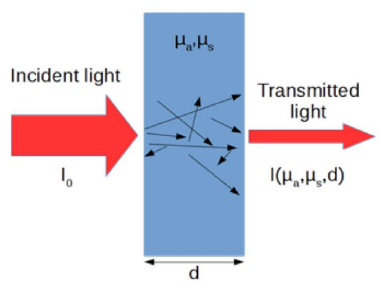

Fig. 2. Optical transmission in heterogeneous media.

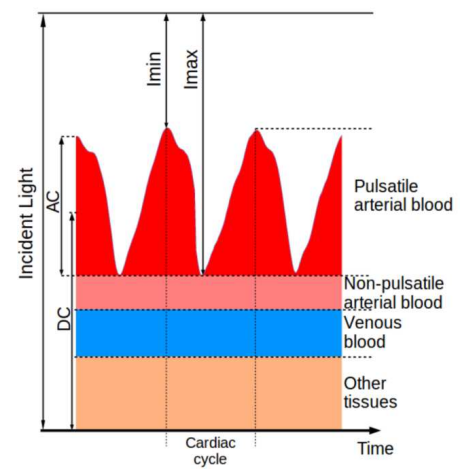

Fig. 4. Absorption of light in tissues (scale not respected, AC component dilated).

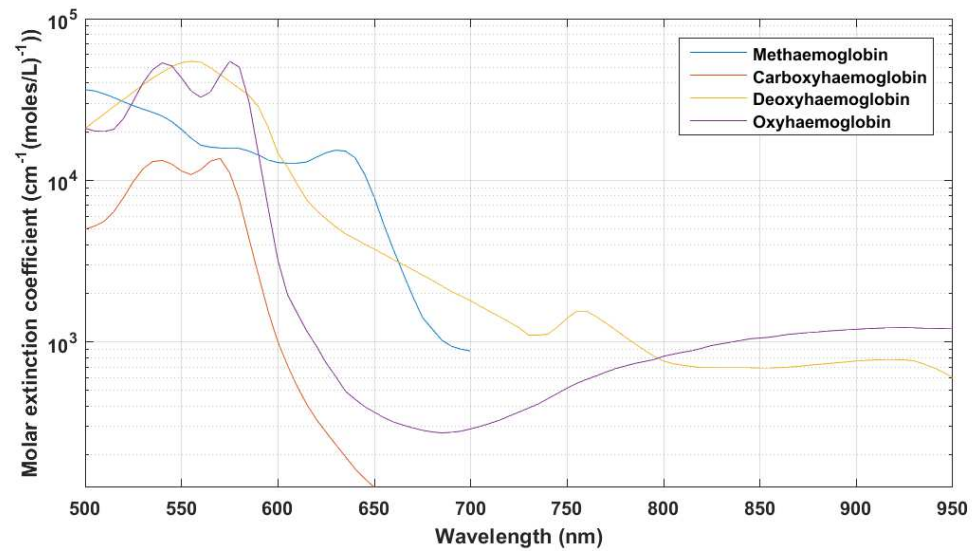

Fig. 3. Molar extinction of various haemoglobin forms. Original data coming from http://omlc.org.
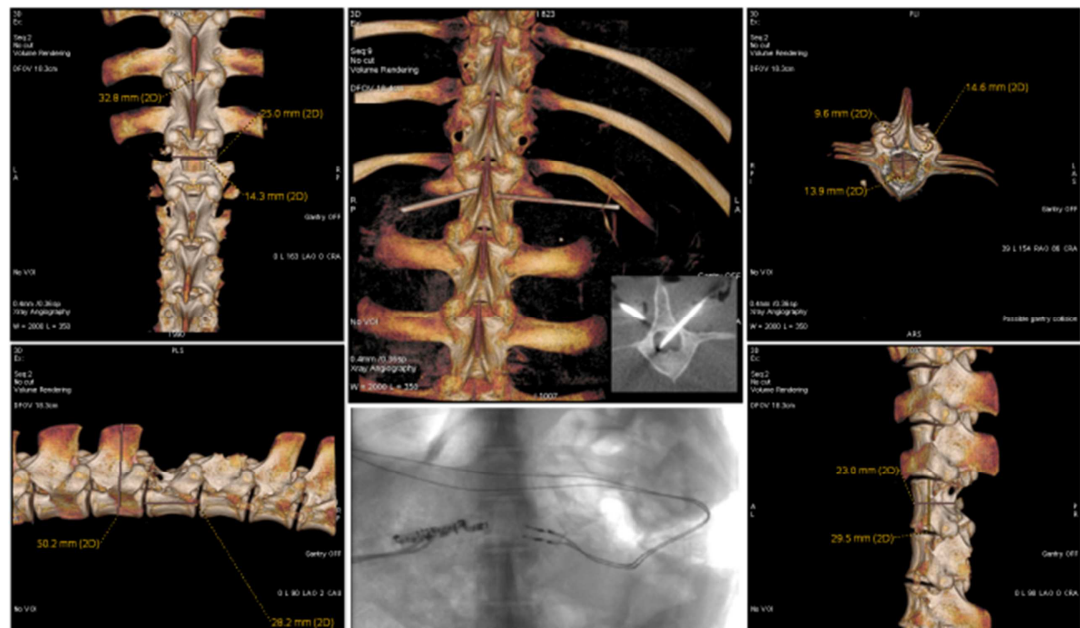

Fig. 5. In vivo $\mathrm{X}$ rays imagery of the pig SC region under study before and after laminectomy. 


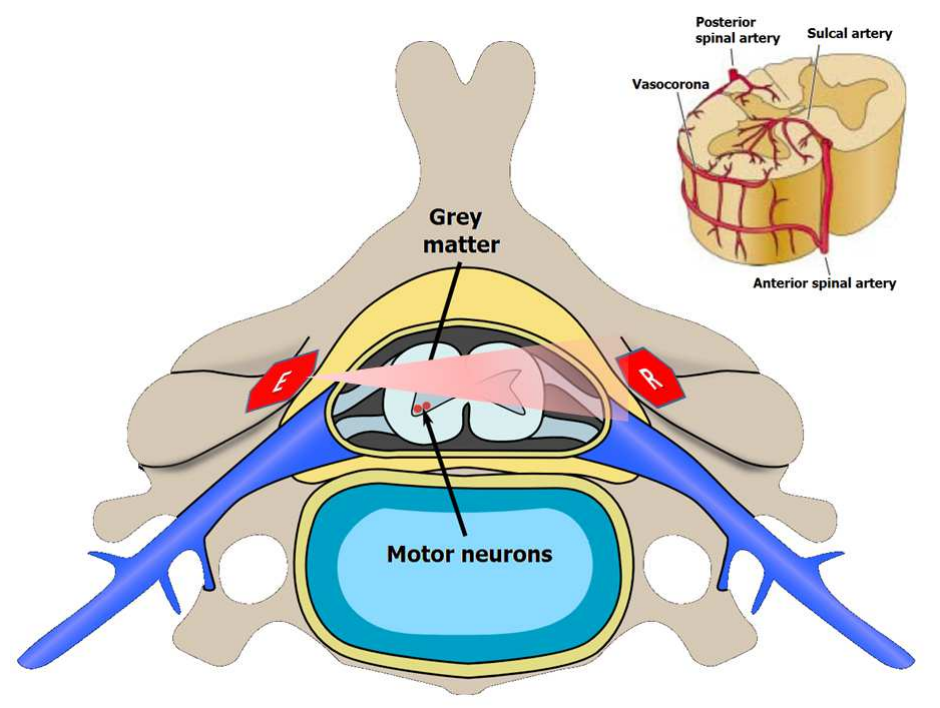

Fig. 6. Illustration of the optical imaging process in vertebra in transmission mode before laminectomy (E means emitter and $\mathrm{R}$ receiver). Original images coming from Wikipedia and http://mskanatomy.blogspot.fr.

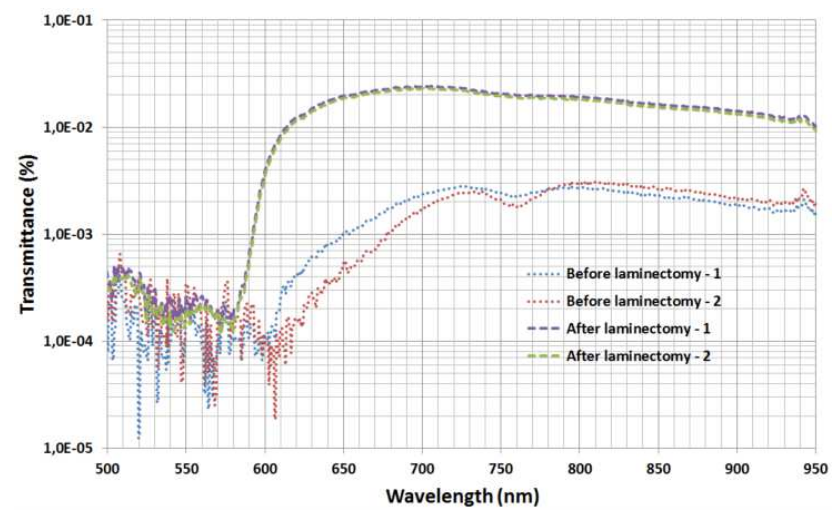

Fig. 8. In vivo transmittance versus wavelength of the SC pig in different conditions.

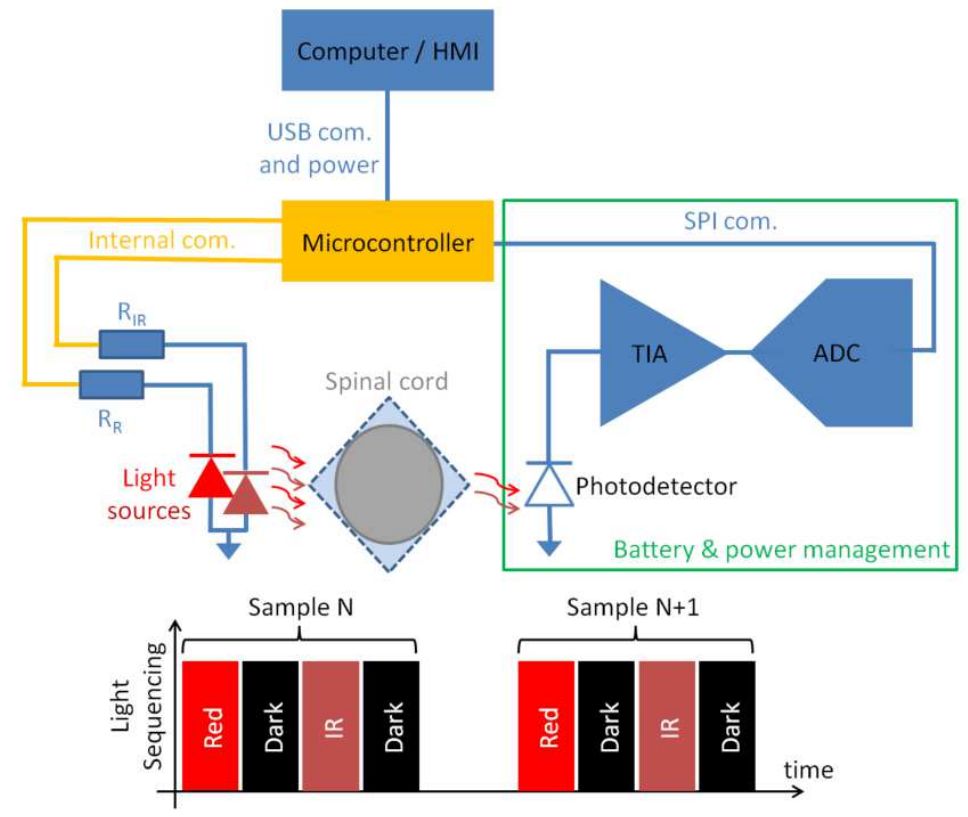

Fig. 7. Synoptic of the opto-electrical part for the SC PPG monitoring, where $\mathrm{ADC}=$ Analog-to-Digital Convertor, TIA $=$ TransImpedance Amplifier, HMI = Human-Machine Interface, with its timing for data acquisition, where dark information is taken between both light illuminations.

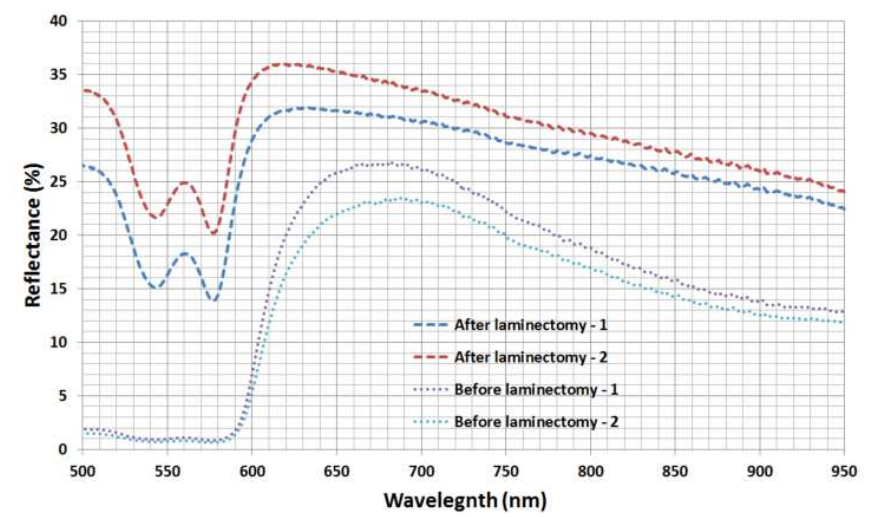

Fig. 9. In vivo reflectance versus wavelength of the SC pig in different conditions. 

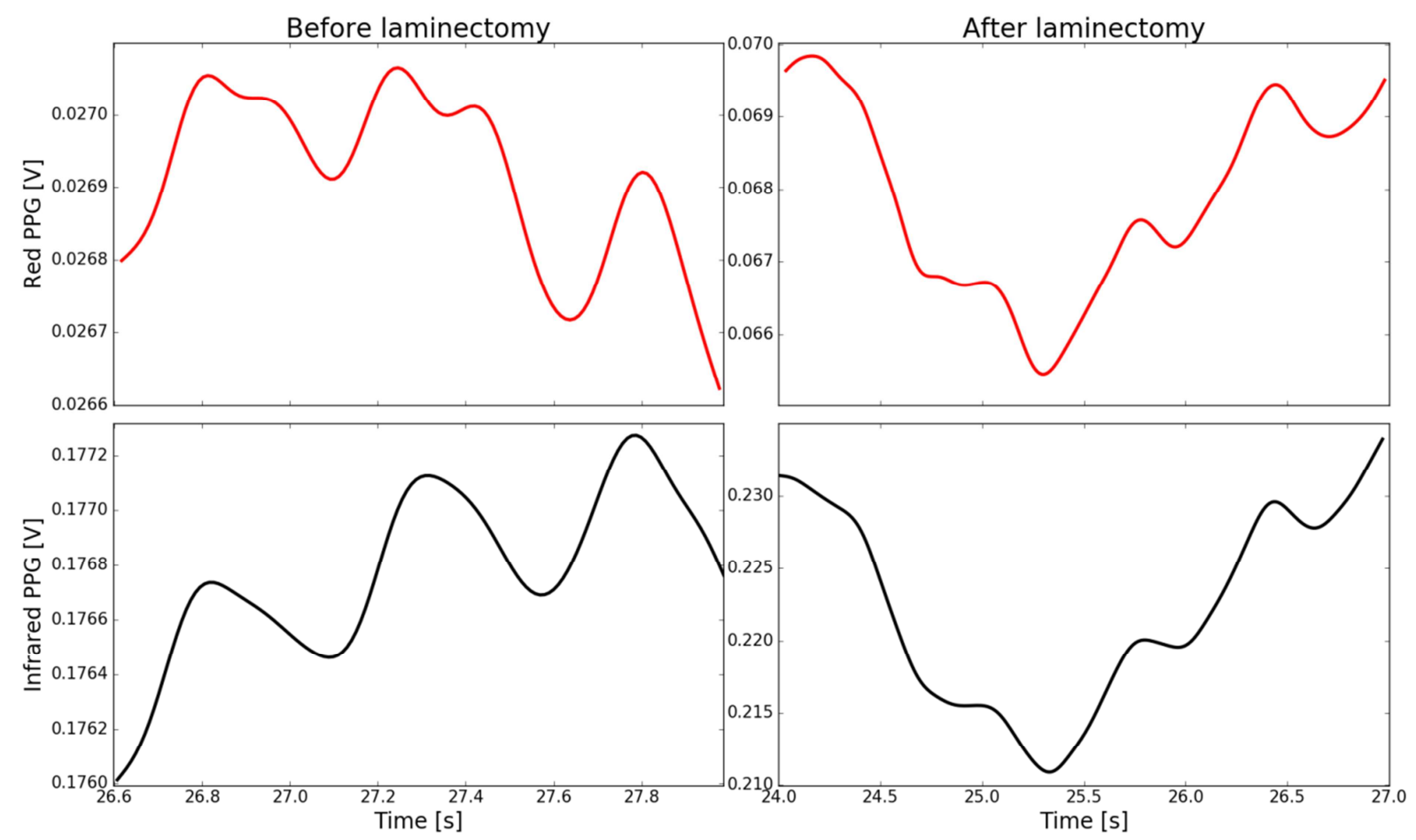

Fig. 10. PPG of the pig SC at the rest, with red and infrared light sources in both cases (case 1 and case 2).

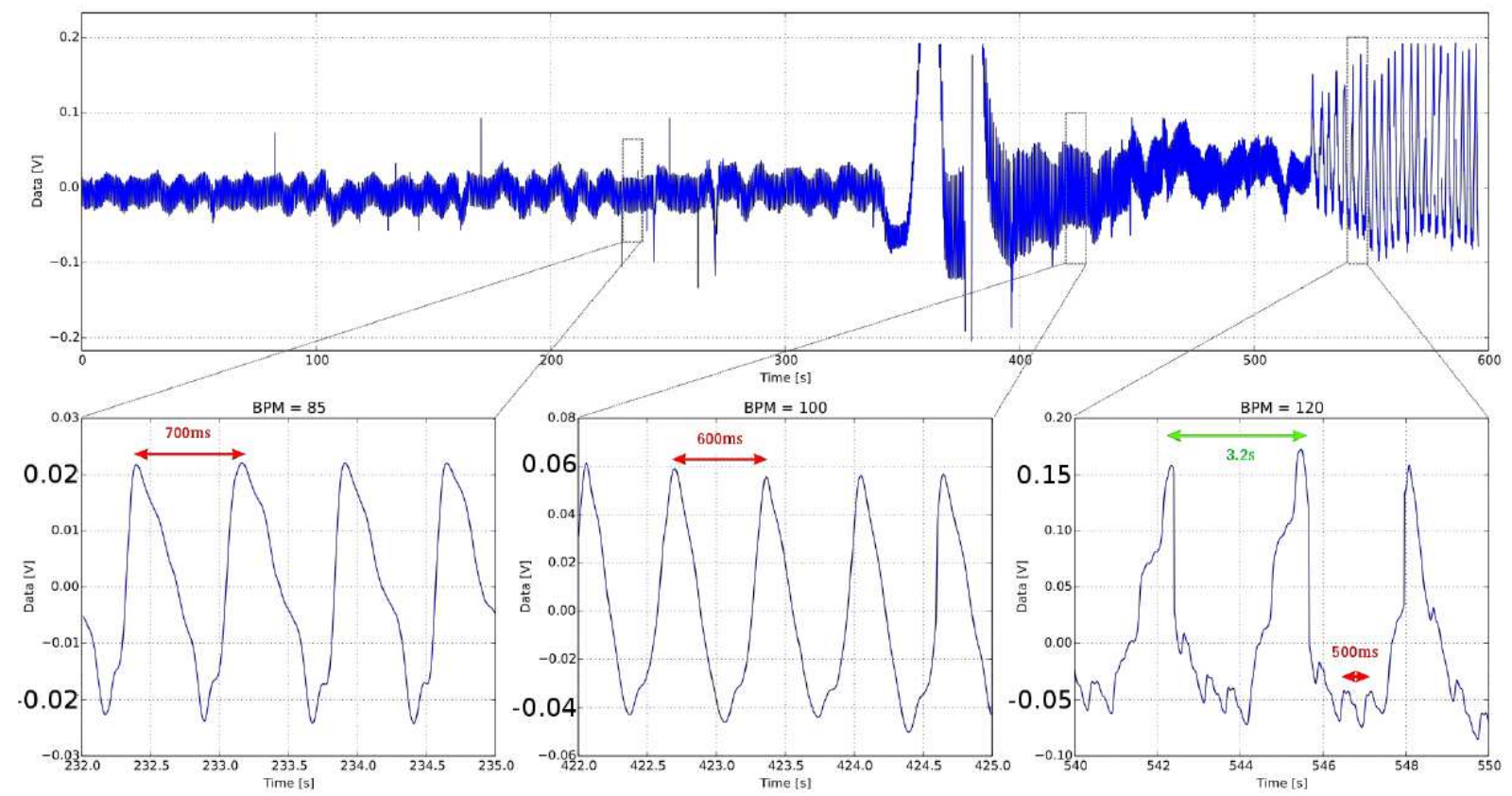

Fig. 11. PPG of the pig SC in case 2, obtained with the IR light source, before and after chemical stimulation. 